\title{
A Spatially-Resolved operando High-Energy Confocal X-ray Diffraction Method for Observing Non-Uniform Degradation Phenomena in a Practical Cylindrical Lithium-ion Battery
}

\author{
Teruhisa BABA, Naoki TAKAO, Yoshitake HONDA, Masazumi ARAO, \\ Masashi MATSUMOTO, Takanori ITOH, and Hideto IMAI*
}

Device Analysis Department, NISSAN ARC Ltd., 1 Natsushima, Yokosuka 237-0061, Japan

*Corresponding author: imai@nissan-arc.co.jp

\begin{abstract}
We report the results of spatially-resolved non-destructive operando electrode reaction analysis for practical cylindrical 18650 battery cells by using a high-energy confocal X-ray diffraction (XRD). A combination of highenergy X-rays ( $72 \mathrm{keV}$ ) and a confocal XRD method, which extracts structural information in a limited area that satisfies a confocal condition, allows us to observe electrode reactions in a cylindrical battery cell in a nondestructive way, resolving the double-side-coating electrode structure.

We observed that electrode reactions were faster in the outer-part electrode than in the inner-part at the initial state reflecting intrinsic cell structure (position of current tab). For a battery cell deteriorated after 500 charge/ discharge cycles, in contrast, electrode reactions were faster in the inner-part electrode than in the outer-part, suggesting that the outer-part is more deteriorated than the inner part. The results of characterization of disassembled electrodes show that the observed slow response of the outer-electrode of a 500-cycled cell is attributed to various factors increasing resistance such as cracks in cathode particles, formation of insulating surface oxide-phase, and anomalous growth of solid electrolyte interphases (SEIs). As shown here, the high-energy confocal XRD is effective for non-destructive analysis of electrode reactions.
\end{abstract}

(c) The Electrochemical Society of Japan, All rights reserved.

Keywords : Lithium-ion Batteries, Confocal X-ray Diffraction, Non-destructive Analysis, Spatially-resolved Analysis

\section{Introduction}

Most electrode reactions and deterioration phenomena occurring in practical Li-ion batteries (LIBs) remain in a black-box. When the LIB cells are disassembled after several hundreds of charge/ discharge cycles, many colored parts are found everywhere in the electrodes, suggesting that electrode reactions and deterioration occur non-uniformly and complexly. ${ }^{1-4}$ Such non-uniform reactions in practical LIB cells considerably impact performance and capacity retention in charge/discharge cycles. The non-uniformity is considered to be ascribed for inhomogeneity of electrodes introduced in manufacturing processes and/or intrinsic structures and arrangement of electrodes in laminated- or wound-LIB cells. ${ }^{4,5}$ The details, however, remain unknown, because few experimental methods can directly observe LIB cells non-destructively under operation.

To date, non-uniformity has been observed for disassembled cells: i.e., electrochemical performances and qualitative and/or quantitative interpretation of electrode states have been evaluated by using small test cells for a chosen local part of disassembled electrode sheets extracted from the cycled practical laminated- or wound-cylindrical cells. Physical and chemical analyses have also been done for disassembled electrodes. In fact, there should be temperature and/or pressure distributions depending on operation. This effect, however, has not been able to be taken into account for disassemble analysis and thus, obtained results might not be same as those for the disassembled cells.

Additionally, much effort has been made to develop nondestructive operando evaluation techniques for electrode reaction analysis. Among them, X-ray absorption spectroscopy, ${ }^{6-9}$ confocal X-ray diffraction (XRD), ${ }^{10,11}$ and neutron diffraction ${ }^{12,13}$ successfully analyze non-uniform reactions in a single electrode level. They are, however, difficult in principle to apply to practical cells that have thick cell housing, and no example has been reported.

In this research, we therefore propose applying the spatiallyresolved confocal XRD with high-energy incident X-rays to solve the above-mentioned issues. Combining high-energy X-rays of $72 \mathrm{keV}$ with high-penetration capability and the spatially-resolved confocal XRD technique, electrode reactions in each electrode sheet in a practical 18650 cylindrical cell were non-destructively observed in a wound battery cell along the diameter direction. We achieved a good spatial resolution of below $20 \mu \mathrm{m}$ and successfully analyzed resolving the structural change in double-side-coating electrodes. We observed non-uniformity of the reactions, i.e., the reaction rate differs depending on electrode positions and the reaction rate is slower for the electrode on the outer-side of a practical 18650 cell after hundreds of cycle tests. The results of additional physical analyses of the disassembled electrodes indicated that the alternation of the surface of cathode materials and growth of solid electrolyte interphase (SEI) layers primarily cause such non-uniformity.

So far, such non-uniform behaviors of electrodes have been able to be observed only by disassembling the cells and reassembling a small test cell from the extracted electrodes. The present high-energy spatially-resolved confocal XRD method allows us to observe true reaction uniformity in large-scale practical cells as it is. This provides a smart analysis option for developing practical batteries.

\section{Experimental}

2.1 High-energy confocal XRD for operando measurements

We adopted confocal XRD as a non-destructive analytical method for practical cylindrical LIBs. Generally, the lattice constants (Bragg angle) of cathode and anode materials change upon lithiation/de-lithation during charge/discharge. By monitoring 
(a)

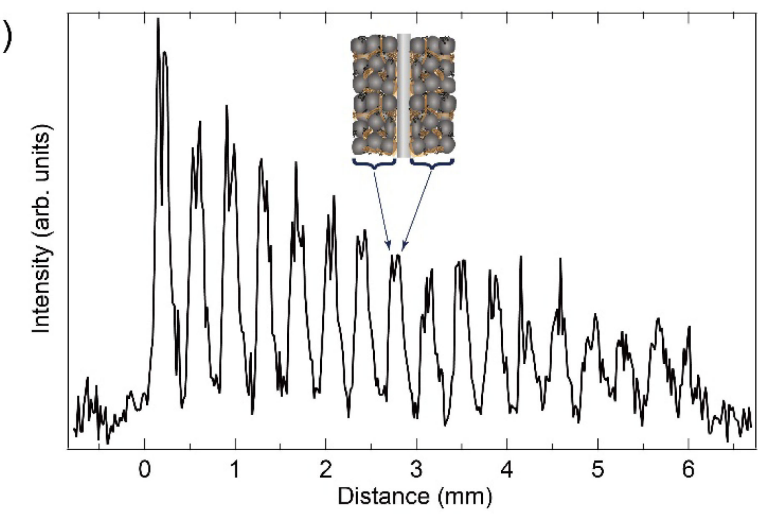

(b)

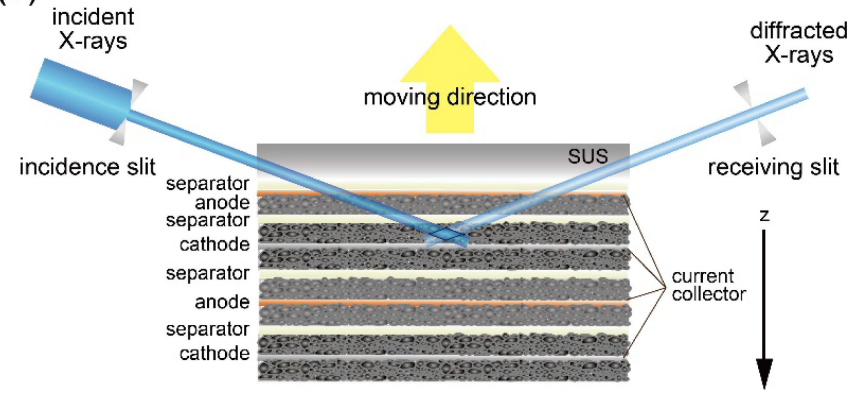

(c)

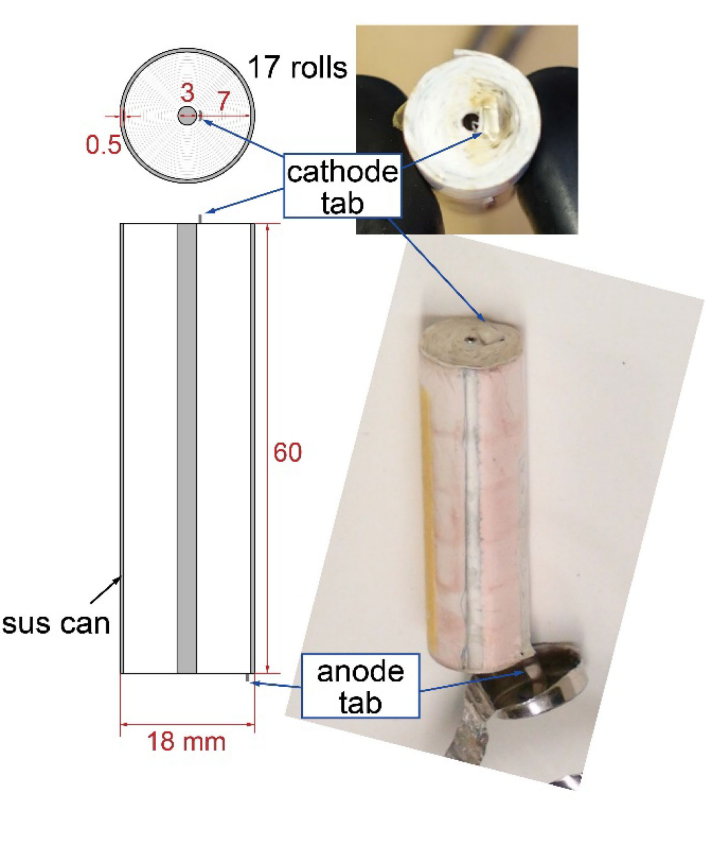

Figure 1. (a) Position dependence of NMC (003) diffraction peak. (b) Schematic image of set-up for confocal XRD. (c) Detailed structure of 18650 cell.

these changes in XRD measurements, we can track and analyze electrode reactions. The confocal XRD method allows spatiallyresolved XRD measurements by measuring Bragg reflections from a confocal point optically defined by collimating slits. In the present experiment, we set the sizes of incidence slit and receiving slit to $40 \mu \mathrm{m}$ and achieved spatial-resolution of $20 \mu \mathrm{m}$.

High-energy X-rays of $72 \mathrm{keV}$ were utilized to obtain diffraction signals from the inner-part of wound cells penetrating the thick housing of a 18650 practical cell. We chose three position along the diameter direction (denoted as "out," "middle," and "in") and observed a (003) diffraction peak of a $\mathrm{Li}\left(\mathrm{Ni}_{1-x-y} \mathrm{Mn}_{x} \mathrm{Co}_{y}\right) \mathrm{O}_{2}$ (NMC) cathode. The diffracted X-ray was detected by a YAP $\left(\mathrm{Ce}^{+}\right.$ doped yttrium aluminum perovskite) scintillation counter. We prepared there practical cells (initial, 200 cycled, and 500 cycled) for operando measurements charging with $1 \mathrm{C}$ rate with a constantcurrent mode. These confocal XRD measurements were performed on a beamline BL16XU at SPring-8.

\subsection{Test cell and discharge/discharge cycle conditions}

The cylindrical 18650 LIB cell $(2.1 \mathrm{Ah})$ used consists of a graphite anode, NMC cathode, and lithium hexafluorophosphate $\left(\mathrm{LiPF}_{6}\right)$ in a mixture solvent of ethylene carbonate $(\mathrm{EC})$, propylene carbonate (PC), ethyl methyl carbonate (EMC), and dimethyl carbonate (DMC) as electrolyte. The cell dimensions are $\phi$ $18 \mathrm{~mm} \times \mathrm{H} 65 \mathrm{~mm}$, and the double-side-coating electrode is wound in the cylindrical cell. The electrode is approximately $80 \mu \mathrm{m}$ thick (single-side of cathode).

Charge/discharge cycles were performed at $25^{\circ} \mathrm{C}$ with $1 \mathrm{C}$ rate up to 500 cycles. The battery cells were charged with a constant current-constant voltage (CC-CV) and discharged with a CC cut-off condition in the range of $2.6-4.2 \mathrm{~V}$. Before and after cycles, we measured the cell capacities at a low rate of $0.1 \mathrm{C}$ and the electrochemical impedance spectra (EIS) at a 50\% depth of discharge (DOD) at $25^{\circ} \mathrm{C}$.

\subsection{Characterization of disassembled electrodes}

After the confocal XRD measurements, the cylindrical battery cells were disassembled and the electrodes were characterized by scanning electron microscopy (SEM), transmission electron microscopy (TEM)-electron energy loss spectroscopy (EELS), X-ray photoelectron spectroscopy (XPS), and electrochemical measurements in order to investigate the details of the observed non-uniform reactions. The cells were disassembled in an argon glove box, and all measurements were performed without exposing the cell to the air by using transfer vessels.

\section{Results and Discussion}

\subsection{High-energy confocal XRD measurement}

First, we demonstrate the ability of high-energy confocal XRD measurements on a practical LIB cell. Figure 1(a) exhibits the position dependence of a NMC (003) diffraction peak. The incident $\mathrm{X}$-rays were scanned from the outer-part of the 18650 cell to the inner part with $20 \mu \mathrm{m}$ steps along the diameter direction (see Fig. 1(b)). In the scanned regions, 17 split diffraction peaks were periodically observed with $380 \mu \mathrm{m}$ intervals. The each split peak reflects the double-side-coating electrode structure, and the number of peaks (electrode sheet) and their intervals fully coincide with the 18650 cell structure (see Fig. 1(c)). This result indicates that electrode states can be monitored by high-energy confocal XRD resolving each electrode layer in double-side-coating electrodes.

\subsection{Operando high-energy confocal XRD measurements}

The charge/discharge cycle dependence of capacity retention of the 18650 cell used in this research is shown in Fig. 2(a). The capacity retention is plotted against the square-root of the cycle number and decreases proportionally to the square-root of the cycle number up to 300 cycles. Beyond 300 cycles, the capacity retention rate drastically deviates from the square-root law. Figure 2(b) shows the EIS spectra. A drastic increase of resistance was observed for the 500-cycled cell.

Next, we show the results of operando high-energy confocal XRD measurements for three 18650 cells (initial, 200 cycled, 500 cycled) charged with $1 \mathrm{C}$ rate. Three points ("out," "middle," and 
Electrochemistry, 88(2), 63-68 (2020)
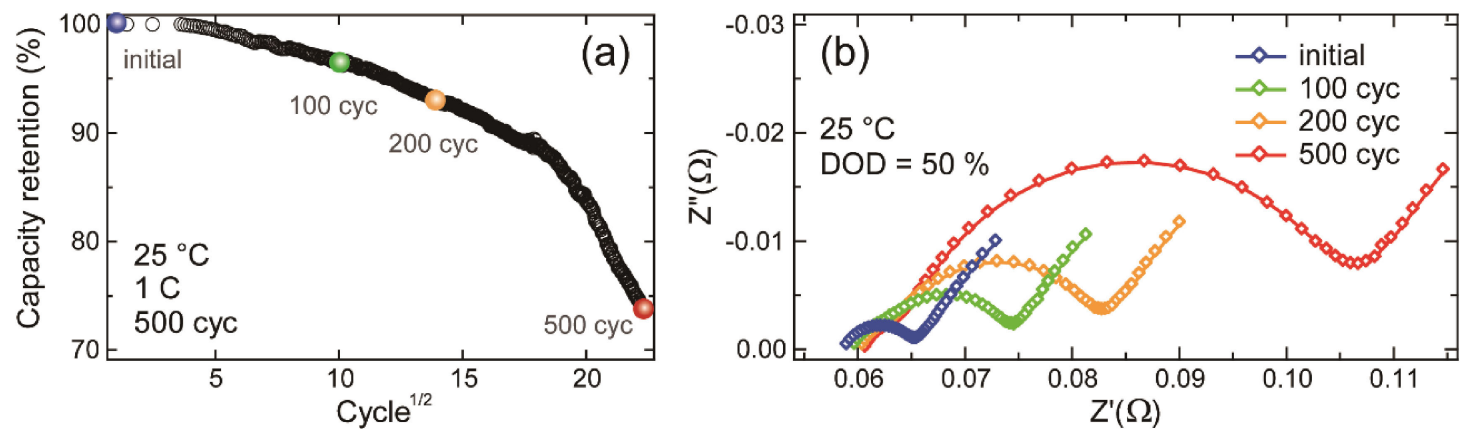

Figure 2. (a) Charge/discharge cycle dependence of capacity retention. (b) Electrochemical impedance spectra.

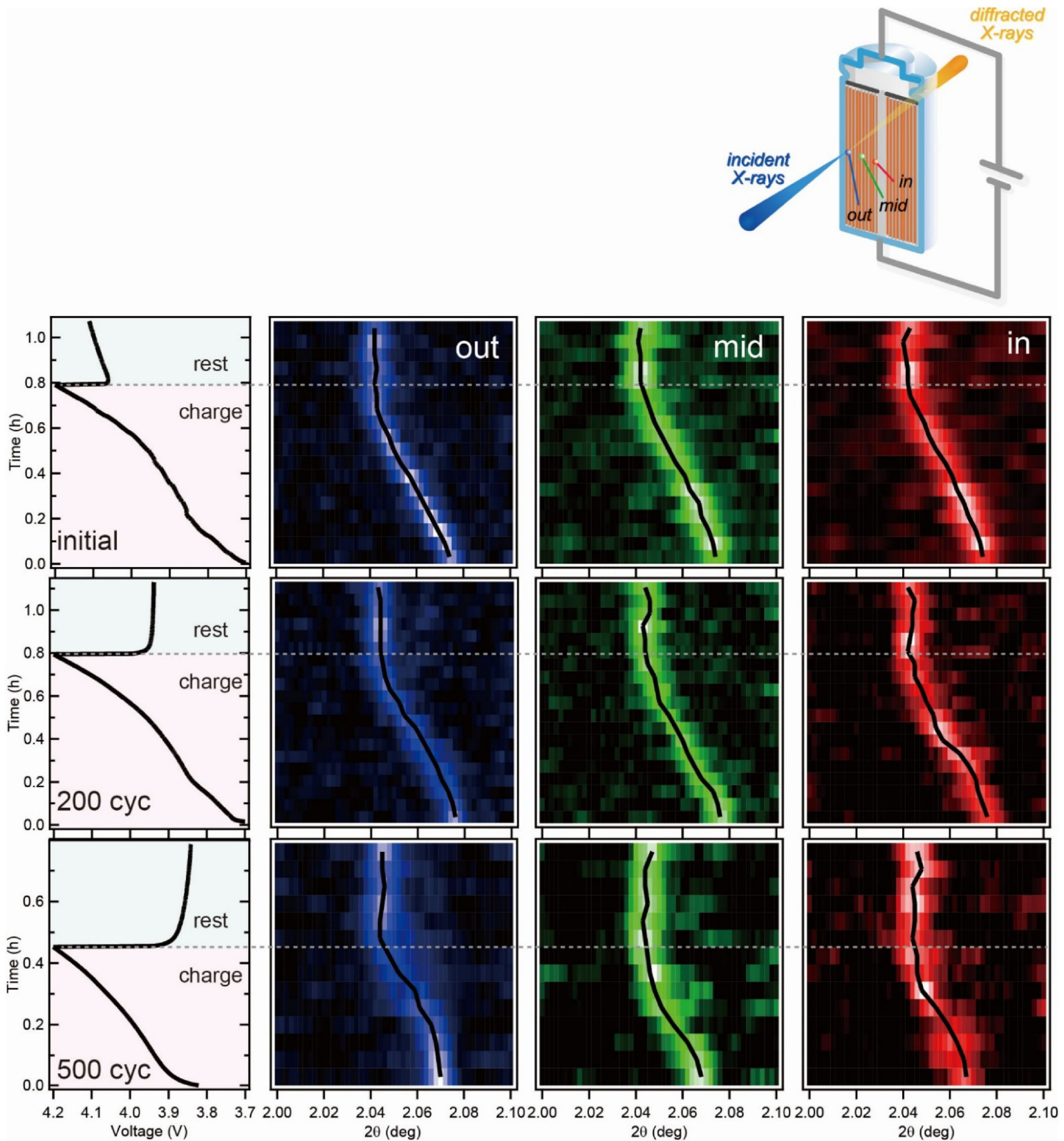

Figure 3. Results of operando high-energy confocal XRD measurements for three 18650 cells (initial, 200 cycled, and 500 cycled). The charge-curves are given in the far left graphs, and changes of NMC (003) diffraction peaks are shown in the three graphs to the right.

"in") were chosen for measurements for each cell. The positions of "out," "middle," and "in" are the second, eighth, and fifteenth electrode sheets from the edge, respectively. The charge-curves are given in the far left graph of Fig. 3, and changes of NMC (003) diffraction peaks are shown in the three graphs to the right. All diffraction peaks shifted to a lower angle upon charging. This result corresponds to an increase in lattice constant in NMC cathodes during charging. 


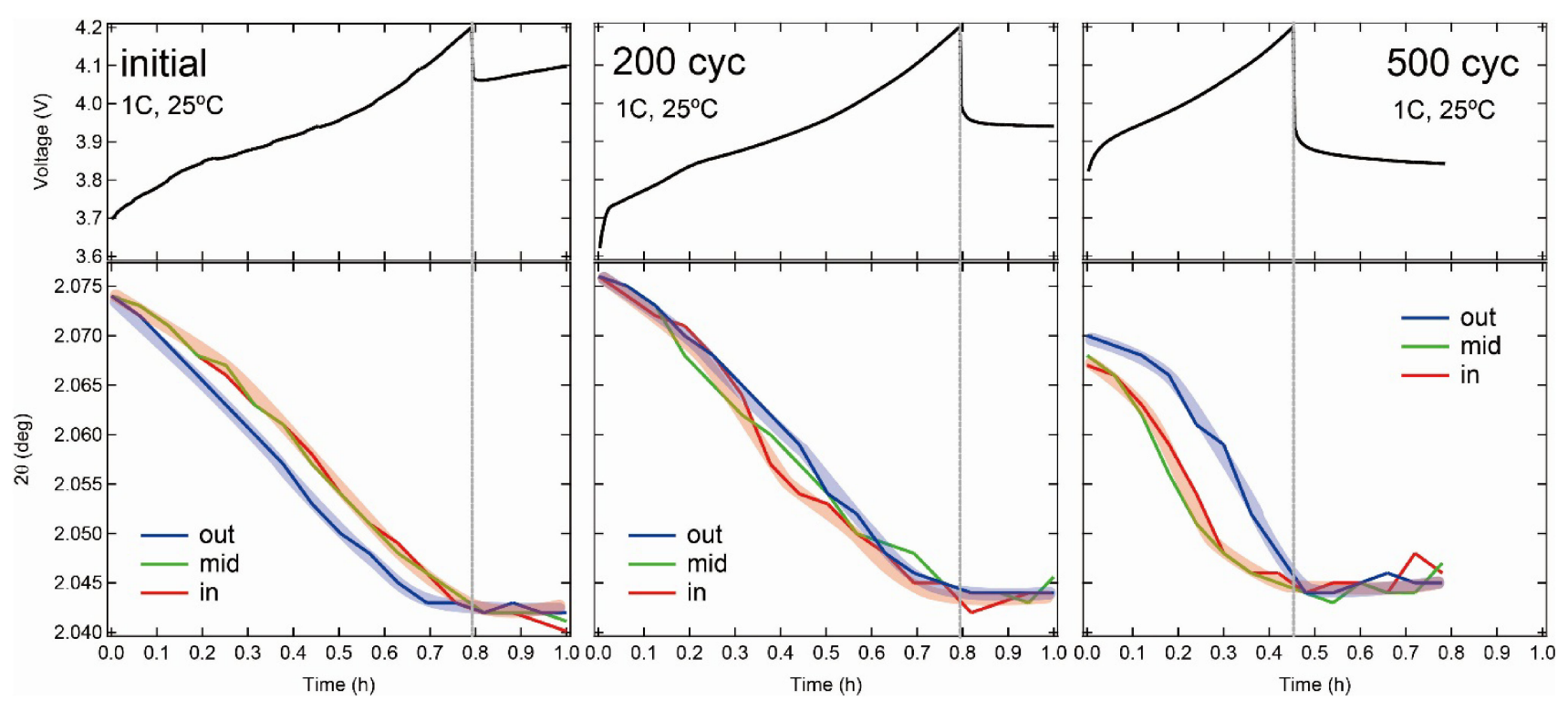

Figure 4. The time variation of diffraction angle during charging for initial, 200-cycled, and 500-cycled cells.

Figure 4 shows the time variation of the diffraction angle during charging for initial, 200-cycled, and 500-cycled cells. For the initial cell, the electrode in the outer part shows fast charging behavior. Since the anode current-tab is arranged at the outer-side in this cell (See Fig. 1(c)), current density during charging was larger in the outer-side, and de-lithiation occurred faster in outer electrodes. For the 200-cycled cell, the structure change of NMC in the electrode proceeds almost at the same time at "out," "middle," and "in" parts. For the 500-cycled cell, reactions occurred slowly in the outer electrode.

So far, such position-dependent local reaction behaviors in practical cells have been able to be grasped only by electrochemical measurements for reassembled electrodes made by disassembling original practical cells. The present high-energy confocal XRD offers a completely non-destructive method for observing local electrode reaction everywhere in practical cylindrical cells. In fact, there should be temperature and/or pressure distributions depending on the operation, but this effect has not been able to be taken into account for reassembled electrochemical tests, so obtained results might not be the same as those of the real cells. From this point of view, the present high-energy confocal XRD is effective to evaluate the practical cells and provides complementary information to electrochemical evaluations.

In the following, we will present the results of various analyses to understand the details of observed battery behaviors.

\subsection{Characterization of disassembled electrodes}

Delays in charge reaction observed in cycled and deteriorated electrodes, especially the outer-part of 500-cycled electrodes, are considered to increase resistance caused for various reasons such as alternations of electrode structures, bulk and/or surface structures active materials, etc.

Figure 5(a) and (b) show the discharge curves of the reassembled coin-type cells made from NMC cathodes from deteriorated cells. The reassembled cells were discharged in a region of $2.6-4.2 \mathrm{~V}$ at a constant current of $0.1 \mathrm{C}$ at $25^{\circ} \mathrm{C}$. We can clearly see that the capacity of outside electrodes of the 500-cycled cell drastically decreases. Figure 5(c) and (d) also show the EIS spectra. We can again see that the resistance for outside electrodes of the 500-cycled electrode drastically increases. These behaviors coincide with operando confocal XRD results.

The results of cross-sectional SEM, TEM observation, and EELS analysis, revealed the formation of cracks in cathode materials and
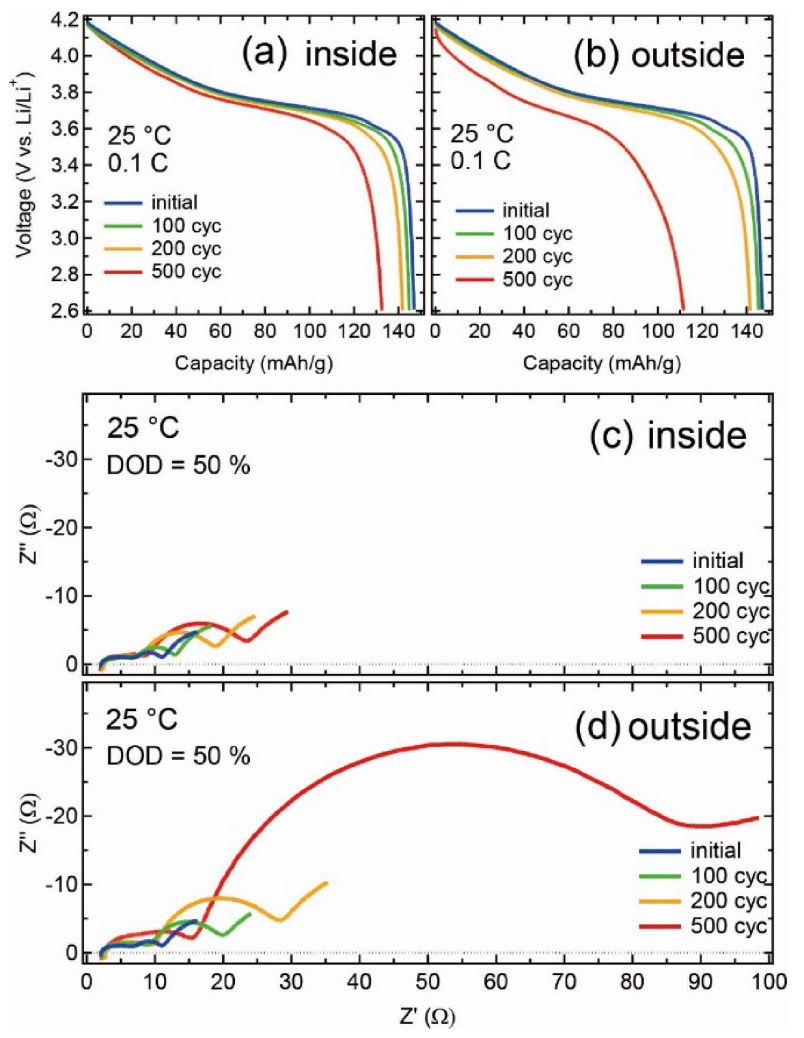

Figure 5. (a) and (b) Discharge curve of reassembled coin-type cells made from NMC cathodes from deteriorated cells in inner- and outer-parts, respectively. (c) and (d) EIS spectra in inner- and outerparts, respectively.

surface structure alternation ascribed for capacity fade and resistance increase. Figure 6(a)-(c) show the cross-sectional SEM images of cathode materials. We can see that cracks were formed in 500-cycled cathode particles, especially at outside electrodes.

The alternations of surface structure of cathode primary particles were confirmed by TEM observation and EELS analysis. As shown in Fig. 7(a) to (c), the surface of particles covered with crystalline phase differs from the layered bulk structure. The thickness of the crystalline phase was increased as charge/discharge cycles increased 

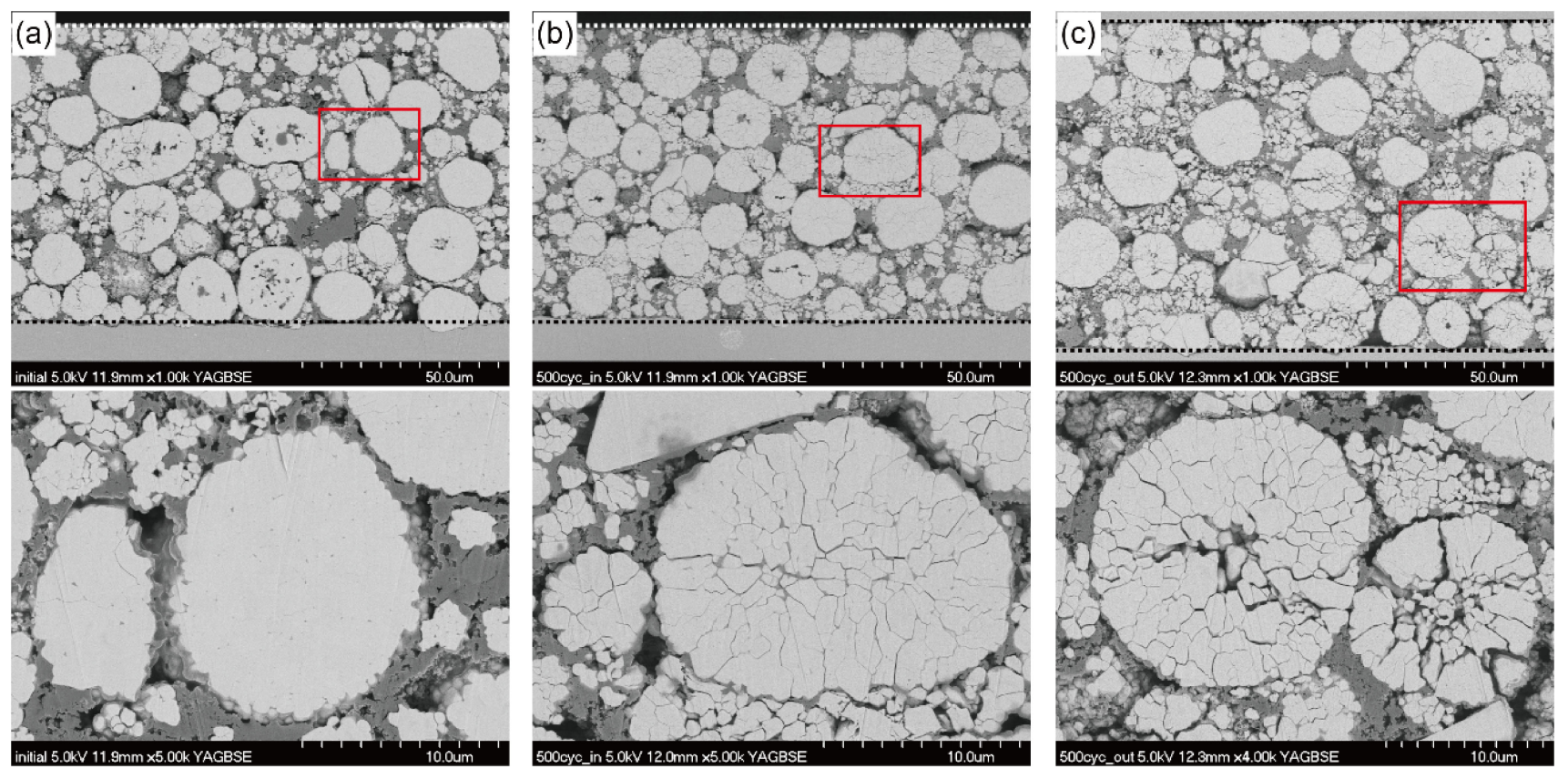

Figure 6. The cross-sectional SEM images of cathode materials. (a) Initial electrodes, (b) 500-cycled electrodes in inner-part, and (c) 500cycled electrodes in outer-part.
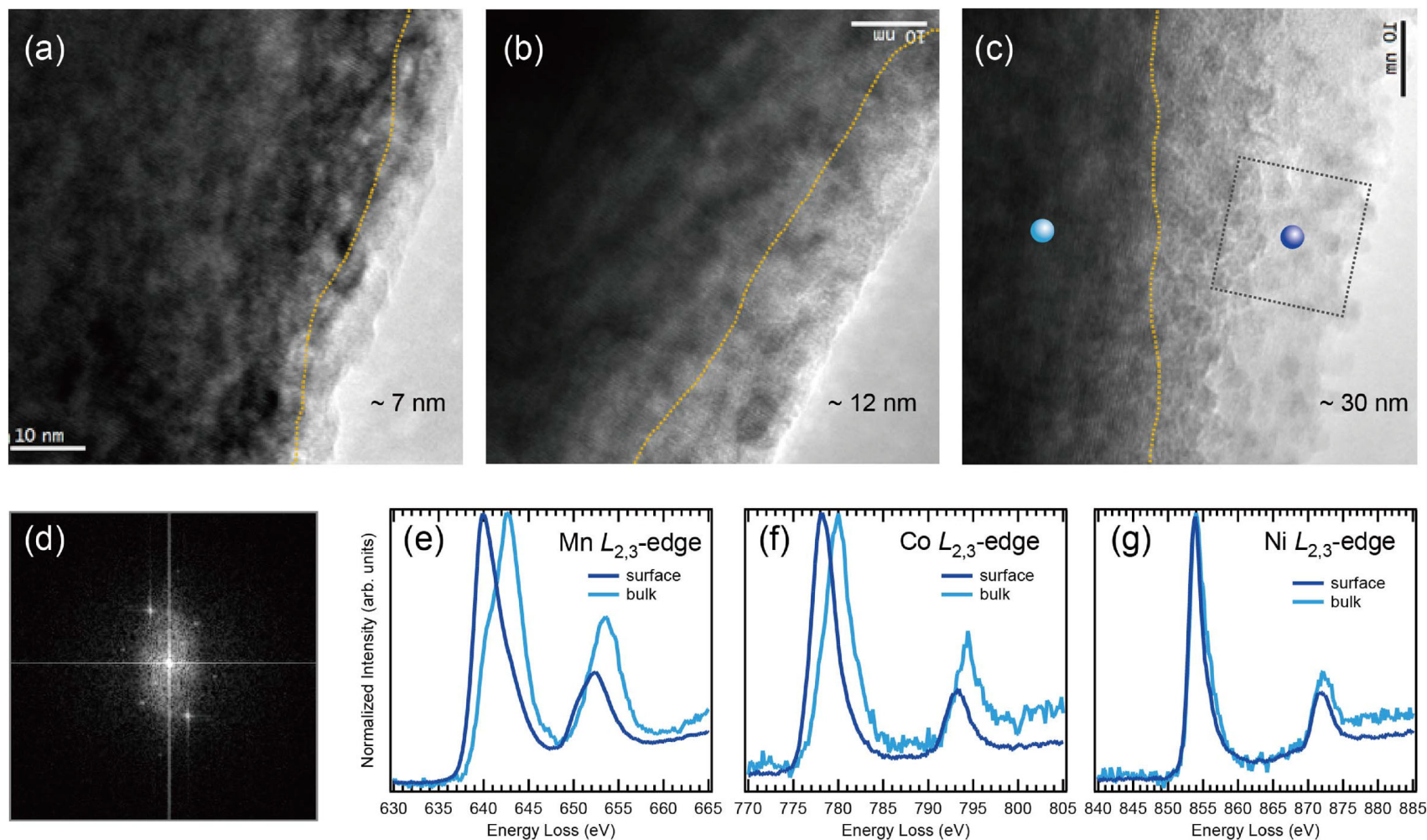

Figure 7. (a) to (c) Cross-sectional TEM images of cathode materials. (a) Initial electrodes, (b) 500-cycled electrodes in inner-part, and (c) 500-cycled electrodes in outer-part. (d) FFT pattern of surface area depicted in Fig. 7(c). (e)-(g) Mn, Co and Ni $L_{2,3}$-edge EELS spectra depicted in Fig. 7(c).

and reached almost $30 \mathrm{~nm}$ for particles in the outer-part in 500cycled electrodes. These surface phases are considered to be rocksalt divalent transition metal oxides, such as $\mathrm{MnO}, \mathrm{CoO}$, and $\mathrm{NiO}{ }^{14}$ Figure 7(d) shows a fast Fourier transform (FFT) pattern of the surface area depicted in Fig. 7(c) and suggests that this surface phase has a $\mathrm{NaCl}$ rock-salt structure. ${ }^{15,16}$ Also EELS spectra of deteriorated samples show an increased portion of low valence states. (Fig. 7(e)-(g)) The electrical conductivity of these surface phases are known to be low, and this can increase resistance for charging. ${ }^{17}$

Another factor for increased resistance and capacity fade is the growth of SEI. Growth of SEI can be evaluated by O $1 s$ XPS spectra. Figure 8(a) and (b) show O $1 s$ XPS spectra for 500-cycled electrodes in the inner- and outer-parts, respectively. The peak 


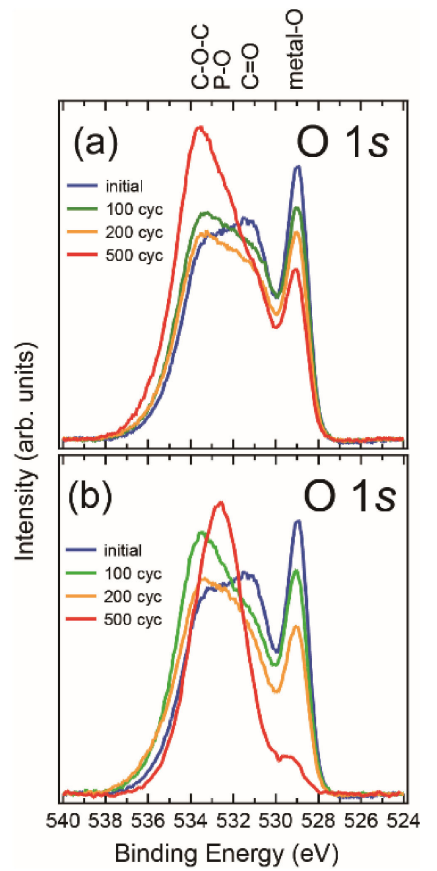

Figure 8. (a) and (b) O $1 s$ XPS spectra for 500-cycled electrodes in inner- and outer-parts, respectively.

centered at $529 \mathrm{eV}$ is attributed to a metal-oxygen (M-O) chemical bond in cathode materials. ${ }^{17}$ By monitoring the intensity of this peak, we can indirectly estimate the thickness of formed SEIs. For both inner- and outer-parts, the peak attributed to M-O bond gradually decreased as charge/discharge cycles increased. Especially, the decrease of the signal is dominant for the outer-part for the 500 -cycled electrode, indicating that SEI grew rapidly at the outerpart for the 500-cycled cell, and this also causes resistance to increase.

As shown above, analyses on disassembled electrodes provide comprehensive structural information to help understand the observed electrode behaviors by non-destructive high-energy confocal XRD. Complementary uses of such non-destructive/ destructive analyses offer an effective experimental methodology to analyze large-scale practical LIB cells.

\section{Conclusions}

In conclusion, by using high-energy X-rays $(72 \mathrm{keV})$ with high penetration capability and by combining it with the staticallyresolved confocal XRD method, we successfully observed nonuniform position-dependent electrode reactions in practical cylindrical 18650 LIB cells in a non-destructive way. The achieved spatial resolution was $20 \mu \mathrm{m}$ in this setup, and double-side-coating electrodes were able to be analyzed.

So far, local electrode reactions have been analyzed by reassembling disassembled electrodes. This is, however, complicated and cannot exactly reproduce true reactions in practical cells by taking effects of temperature and/or pressure distribution that are specific for the practical cells into consideration. Thus, the spatiallyresolved and non-destructive confocal XRD method is highly effective for operando analysis of practical LIB cells.

In this research, we analyzed a deteriorated 18650 cell by highenergy confocal XRD and found that electrode reactions are slower in the outer-part than in the inner part. The results of SEM, TEM, EELS, and XPS on disassembled electrodes revealed the generation of cracks in cathode materials, formation of insulating surface-oxide phases, and anomalous growth of SEIs, which are the main causes of the observed delay in electrode reactions.

Cutting down the abnormal behaviors by a non-destructive operando method and adding complementary analysis for disassembled electrodes, we are able to deeply and effectively understand true electrode states in a practical LIB cells. We expect this method will be useful to increase the performance, lifetime, and safety of practical batteries.

\section{Acknowledgments}

Synchrotron experiments were performed under the approval of the Japan Synchrotron Radiation Research Institute (JASRI). Proposal Nos. 2015B5090, 2016A5092, 2016A5093, 2016B5090, 2017A5090, 2017A5091, 2017B5090, 2017B5091, 2018A5090, 2018A5091, 2018B5091, and 2018B5092.

\section{References}

1. P. D. Prezas, L. Somerville, P. Jennings, A. McGordon, J. Basco, T. Duong, and I. Bloom, SAE Technical Paper, 2016.

2. L. Somerville, J. Bareño, S. Trask, P. Jennings, A. McGordon, C. Lyness, and I. Bloom, J. Power Sources, 335, 189 (2016).

3. C. R. Birkl, M. R. Roberts, E. McTurk, P. G. Bruce, and D. A. Howey, J. Power Sources, 341, 373 (2017)

4. F. B. Spingler, W. Wittmann, J. Sturm, B. Rieger, and A. Jossen, J. Power Sources, 393, 152 (2018).

5. D. Mohanty, E. Hockaday, J. Li, D. K. Hensley, C. Daniel, and D. L. Wood, III, J. Power Sources, 312, 70 (2016).

6. Y. Orikasa, Electrochemistry, 84, 524 (2016) [in Japanese]

7. M. Katayama, K. Sumiwaka, R. Miyahara, H. Yamashige, H. Arai, Y. Uchimoto, T. Ohta, Y. Inada, and Z. Ogumi, J. Power Sources, 269, 994 (2014).

8. Y. Orikasa, Y. Gogyo, H. Yamashige, M. Katayama, K. Chen, T. Mori, K. Yamamoto, T. Masese, Y. Inada, T. Ohta, Z. Siroma, S. Kato, H. Kinoshita, H. Arai, Z. Ogumi, and Y. Uchimoto, Sci. Rep., 6, 26382 (2016).

9. T. Nakamura, T. Watanabe, Y. Kimura, K. Amezawa, K. Nitta, H. Tanida, K. Ohara, Y. Uchimoto, and Z. Ogumi, J. Phys. Chem. C, 121, 2118 (2017).

10. H. Murayama, K. Kitada, K. Fukuda, A. Mitsui, K. Ohara, H. Arai, Y. Uchimoto, Z. Ogumi, and E. Matsubara, J. Phys. Chem. C, 118, 20750 (2014).

11. K. Kitada, H. Murayama, K. Fukuda, H. Arai, Y. Uchimoto, Z. Ogumi, and E. Matsubara, J. Power Sources, 301, 11 (2016).

12. L. Cai, K. An, Z. Feng, C. Liang, and S. J. Harris, J. Power Sources, 236, 163 (2013).

13. H. Zhou, K. An, S. Allu, S. Pannala, J. Li, H. Z. Bilheux, S. K. Martha, and J. Nanda, ACS Energy Lett., 1, 981 (2016).

14. M. Shikano, H. Kobayashi, S. Koike, H. Sakaebe, Y. Saito, H. Hori, H. Kageyama, and K. Tatsumi, J. Power Sources, 196, 6881 (2011).

15. S.-K. Jung, H. Gwon, J. Hong, K.-Y. Park, D.-H. Seo, H. Kim, J. Hyun, W. Yang, and K. Kang, Adv. Energy Mater, 4, 1300787 (2014).

16. H. Kuriyama, H. Saruwatari, H. Satake, A. Shima, F. Uesugi, H. Tanaka, and T. Ushirogouchi, J. Power Sources, 275, 99 (2015).

17. S. Watanabe, M. Kinoshita, T. Hosokawa, K. Morigaki, and K. Nakura, J. Power Sources, 258, 210 (2014). 